\title{
Esthetic Rehabilitation of A Peg-Shaped Lateral Incisor with Ceramic Veneer: About A Case Report
}

Mohsen Maaned ${ }^{1}$, Rihab Dakhli ${ }^{2 *}$, Meriem Ben Miled ${ }^{1}$, Hayet Hajjami ${ }^{3}$, Mounir Cherif ${ }^{3}$

\author{
${ }^{1}$ Resident, Department of Fixed Prosthodontics, Dental Faculty of Monastir, University of Monastir, Tunisia \\ ${ }^{2}$ Associate Professor, Research Laboratory of Occlusodontics and Ceramic Prosthesis LR16ES15, Faculty of Dental Medicine, \\ University of Monastir, Monastir, Tunisia \\ ${ }^{3}$ Professor, Department of Fixed Prosthodontics, Faculty of Dental Medicine, University of Monastir, Monastir, Tunisia
}

DOI: $\underline{10.36347 / \text { sjds.2021.v08i03.002 }}$

| Received: 18.02.2021 | Accepted: 02.03.2021 | Published: 05.03.2021

*Corresponding author: Rihab Dakhli

Abstract

The increasing demands for esthetics and recent advances in esthetic dentistry have led to the development of newer materials and techniques with minimal invasion and better natural appearance. The adhesive dentistry and ceramic veneers allowed all ceramic-bonded restorations to become an esthetic and functional approach to reestablish the smile harmony. This clinical report describes a treatment approach for restoring the esthetics of peg-shaped lateral maxillary incisor with a lithium dicilicate veneer.

Keywords: Peg-shaped incisor, esthetics, ceramic veneers, lithium disilicate.

Copyright $(\mathcal{C} 2021$ The Author(s): This is an open-access article distributed under the terms of the Creative Commons Attribution 4.0 International License (CC BY-NC 4.0) which permits unrestricted use, distribution, and reproduction in any medium for non-commercial use provided the original author and source are credited.

\section{INTRODUCTION}

Microdontia is a tooth size abnormality that can be isolated or part of a genetic syndrome. The most common isolated form is that of the lateral maxillary incisors, known as conoid, "rice-grain" or "peg-shaped" incisors. It is defined as a tooth with a mesio-distal incisal width of the dental crown less than the cervical width. It is also defined as the stop in the development of one or more teeth. This anomaly is common, according to a July 2013 study in the "American Journal of Orthodontics and Dentofacial Orthopedics", the overall prevalence of conoid permanent maxillary lateral incisors was estimated at $1.8 \%$ [1]. The rates are higher in Asian patients $(3.1 \%)$ than in black (1.5\%) and white $(1.3 \%)$ patients. Women are 1.35 times more likely than men to have this abnormality. It can be uni or bilateral with equal prevalence, but is mainly found in the left conoid incisor when only one is affected.

Diagnosis is made by simple visual clinical observation and radiographic examination. It results in reduced tooth size, anterior diastema and, consequently, an unpleasant smile. This is a reason for frequent consultation in order to harmonize the smile and correct the shape of the miniature laterals. The restoration of the conoid incisor, like any restoration, must respect the therapeutic gradient concept [2].The treatment options are numerous: direct restoration with composite resins, composite or ceramic veneers or full coverage crown. Indeed, the development of adhesive and minimally invasive dentistry has made ceramic veneers as an attractive aesthetic and functional approach.

The objective of this clinical report is to describe a conservative treatment approach to restore aesthetic disharmony caused by a lateral peg-shaped incisor.

\section{CASE REPORT}

A 25-year- old patient was looking for a treatment to resolve an. A lithium disilicate veneer was planned. It harmonized the patient's smile, recovering the size, shape and anatomical characteristics of the lateral incisor.

A 25-year-old female patient attended the dental clinic with a chief complaint about the aesthetic disharmony in her smile due to the form and shape of her upper right lateral incisor \#12.

The intraoral clinical examination revealed a disharmonious smile caused by unilateral peg-shaped incisor (Figure-1).

The patient had good periodontal health and a stable intercuspal position, normal vertical and 
Mohsen Maaned et al., Sch J Dent Sci, Mar, 2021; 8(3): 94-97

horizontal overlap, and canine-protected guidance. Clinical examination and radiography survey demonstrated adequate and healthy tooth structure, bone support, and the absence of periapical pathologies. One study cast was made as a supplementary tool to enhance the diagnostic stage.
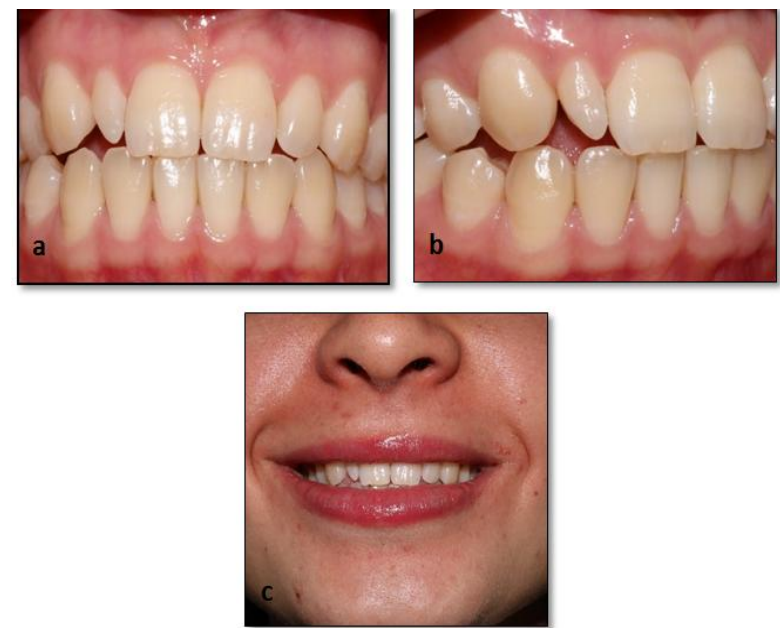

Fig-1: Initial situation (a) intraoral view of the patient's dental status, (b) close-up of the peg-shaped upper lateral incisor, (c) The initial appearance of patient's smile

To simulate the teeth shape design and enhance the patient understanding of proposed treatment plan, a wax setup was made in the study cast (Figure-2). After checking the mesio-distal distance of the pegged lateral incisor with the contralateral one, a conservative treatment using lithium disilicate veneers was planned.

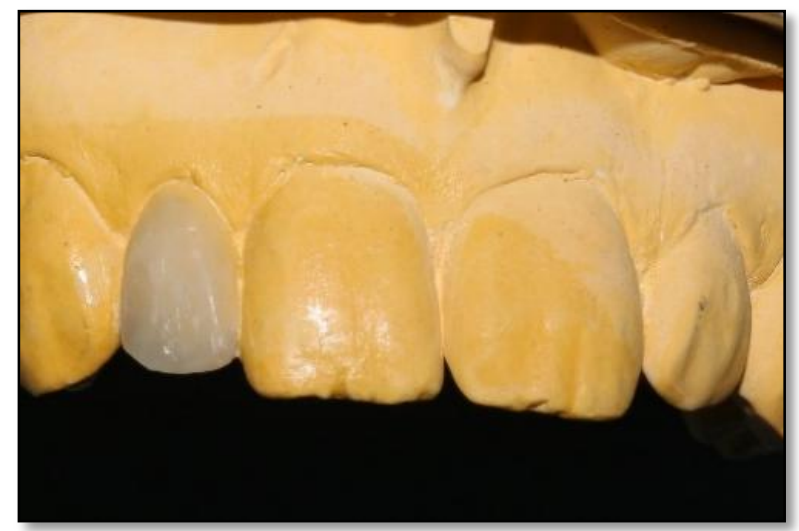

Fig-2: Diagnostic wax-up

The tooth preparation was done restricting entirely to enamel with uniform reduction in buccal surface using a tapered Cylinder Round End Diamond Bur. A small round bur is used to delimit the cervical margin. This preparation was guided by mock-up. The incisal edge was slightly prepared. In this case, the palatal position of the tooth was promising to respect the gradient therapeutic concept and avoid the indication of full coverage crown. Then, a master impression was made using light and heavy silicone Type A (Figure-3).

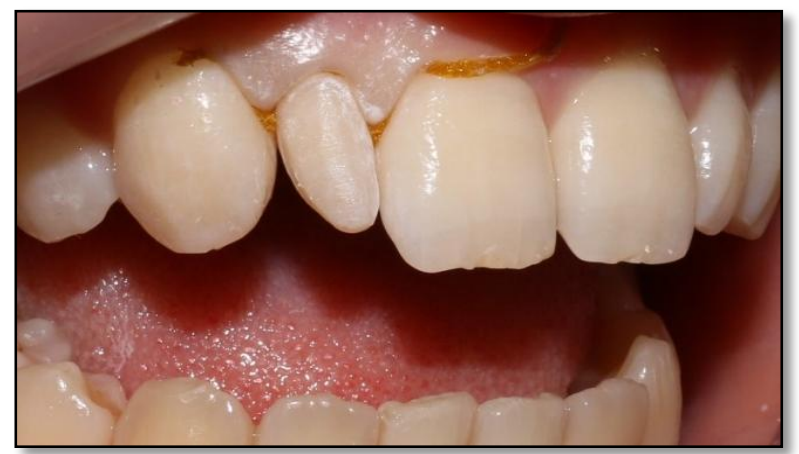

Fig-3: Minimally invasive preparation restricted on the buccal surface

The diagnostic wax setup was used too to perform a silicone key for the fabrication of a provisional restoration fixed with punctual bonding to enhance its stability and to avoid the alteration of the tooth surface (Figure-4).

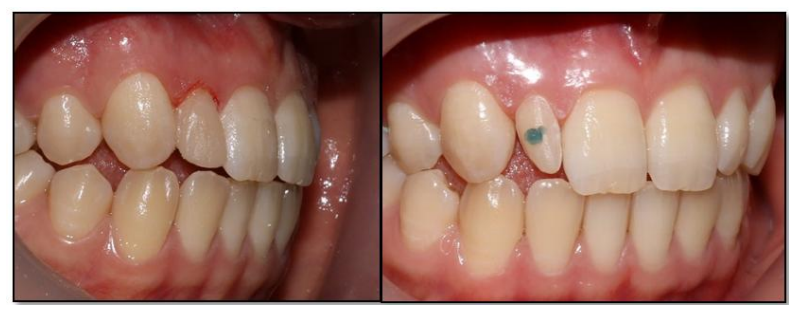

Fig-4: The chair-side provisional restoration fixed with punctual bonding after making a punctual etching

After CAD/CAM procedures, the restoration was received to start the bonding protocol (Figure-5). First of all, marginal adaptation, shape and color were checked, then different try in paste were used to select the suitable shade of the resin material. Since the restoration was made with glass ceramic, so bonding consist on treating both its internal surface and the dental surface.

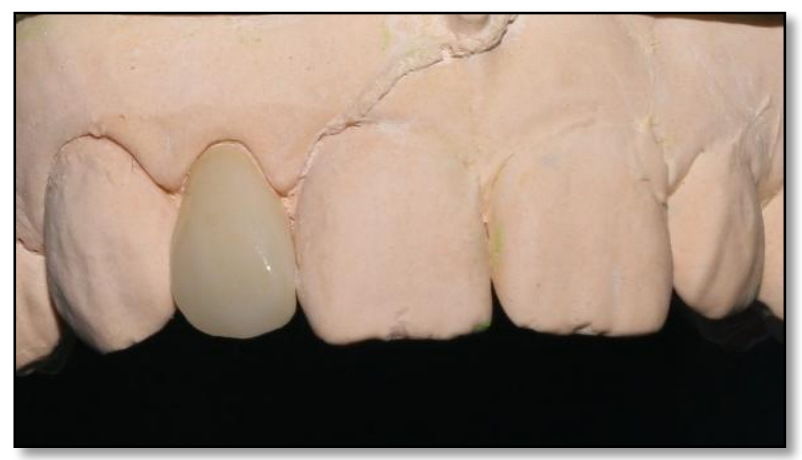

Fig-5: Lithium disilicate veneer

Once the external surface was protected, the internal surface was etched with hydrofluoric acid for 
60 seconds, rinsed, air-dried then a thin layer of a silane agent coupling was applied (Figure 6b).

It is important, to guarantee a good isolation that is why a rubber dam was placed once the preparation was cleaned and polished (Figure 6a). After that, enamel was etched with $37 \%$ phosphoric acid for 30 seconds then rinsed and dried. Finally, bonding system was applied and light cured first, for 5 seconds to remove easily the excess then for 60 seconds at least. The patient was very satisfied with her nice smile (Figure-7).

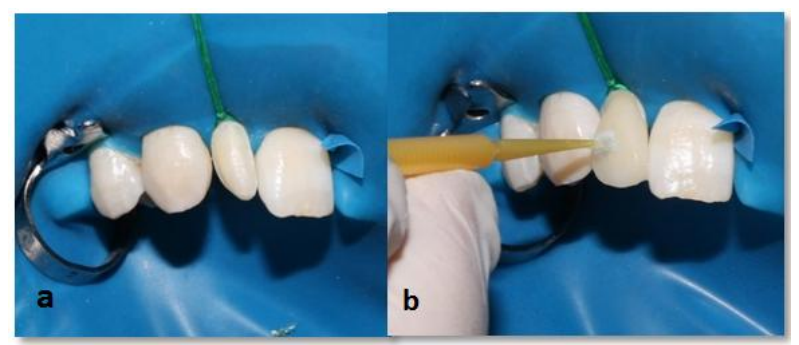

Fig-6: Bonding procedure (a) Rubber dam was placed for a good isolation (b) removal of the excess of luting materiel using a micro brush

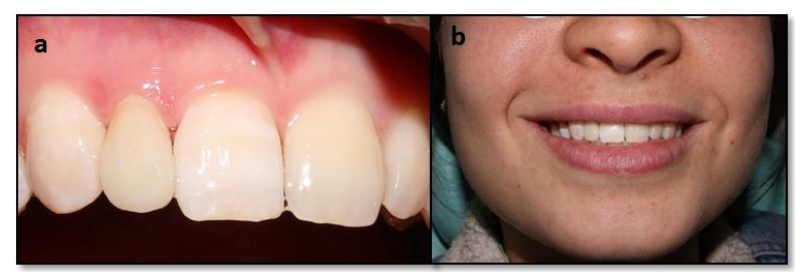

Fig-7: Final Result (a) Close-up of the veneer in position after gentle removal of the resin cement excess, (b) final appearance of the patient showing harmonious smile

\section{DISCUSSION}

According to a meta-analysis, the frequency of maxillary peg-shaped lateral incisors is about $1 \%$ in the general population and up to $1.8 \%$ of the population. Worldwide, 1 in 55 people would be affected by pegged maxillary lateral incisors. The prevalence of unilateral and bilateral pegged lateral incisors is approximately the same. However, when it is unilateral, the left side would appear to be affected twice as much as the right side. In addition, people with Peg-shaped lateral maxillary incisor have a 55\% chance of having contralateral agenesis and can be associated with dental transpositions in $23 \%$ of cases $[1,3]$.

In the clinical situation reported, this abnormality occurs in a rare form affecting the right lateral incisor.

The morphology of the pegged lateral incisor leads us to reflect on the clinical impacts that need to be taken into account for prosthetic rehabilitation. In some cases where patients present a diastema associated with other types of dental malformations (agenesis, malposition...), a combination of different therapeutic options will be necessary. Usually, orthodontics will be needed, sometimes tooth whitening to harmonize the color and the smile and restorative treatment. The strategy is to start with the least invasive solution and then evaluate the result with the patient to approve the suitable approach.

Thus, the aesthetic treatment of the peg-shaped incisor includes the anatomy correction of malformed teeth and the closure of diastema spaces because in such a situation, a central or lateral diastema is frequently diagnosed due to the mismatch between the size of the teeth and the space available on the arch.

However, in this clinical case, the patient had no diastema. Therefore, the objective was just to correct the anatomy of the pegged tooth. Several therapeutic approaches could be considered to resolve such a problem. The restorative ones favors the direct composite restorations as it is conservative, faster and safer treatment. However, color stability and polish retention are some limitations of resin composite restorations in long-term esthetic areas. Likewise, a two year clinical trial had demonstrated that composite veneers were susceptible to chipping when the lateral incisor are restored about 52\% [4-6]. Therefore, ceramic veneer seems to be a best solution to avoid all the disadvantages of composite veneers [7].

Full coverage crowns can be described as an efficient option to correct peg-shaped teeth. Ceramic crowns can perfectly boast the aesthetic result but the full preparation of a pegged lateral have to be well considered on the mechanical and functional components [8]. The indication of the ceramic veneer to treat this case was basically founded on the important size of the tooth. Contrary to small tapered peg-shaped incisors, this pegged lateral offers a wide foundation for the veneer with minimal or none teeth preparation. Thus, the veneer is well reinforced with no risk of cantilevered ceramic.

Another important factor for the simple resolution of this case is that the patient had perfect gingival health and alignment. Given that, in many cases, corrections of the gingival alignment are necessary. As often, the combination of restorative, orthodontic and periodontal techniques is essential to obtain a beautiful and functional result [9].

The different treatment options must be carefully explained to the patient, including the advantages and limitations of each technique, which will help him in the choice of the treatment. In the case presented here, the patient approve with the practionner the indication of indirect ceramic veneer, considering that, this treatment would give predictable esthetic results with minimal or no dental preparation. To help the patient choose the most appropriate treatment, study cast models and diagnostic wax-up were helpful to 
improve the evaluation of anatomical features, occlusion analysis and restoration design, helping the patient to visualize the expected esthetic result.

With a view to adhesion, a total-etch adhesion system was used. These adhesive systems produced high bond strength values when bonded to enamel [10]. In addition, thanks to the palatal position of the tooth, the entire adhesion area was located within the enamel without exposure of the dentin; long-term results may be more favorable once the adhesion to the enamel is stronger and more stable than the adhesion to the dentin.

\section{CONCLUSION}

When it is about restoring peg-shaped lateral incisor, there is no perfect solution to opt. In fact, several therapeutic approaches as direct composite veneer, ceramic veneer or full coverage crown could be considered depending on the clinical situation and the occlusion. Nevertheless, aside this, the approval and the availability of the patient should be taken to account. Ceramic veneer considered as a simple and conservative treatment can lead to a higher esthetic result with functional performance, producing patient satisfaction.

Conflict of Interests: The authors declare that there is no conflict of interests regarding the publication of this paper.

\section{REFERENCES}

1. Hua F, He H, Ngan P, Bouzid W. Prevalence of peg-shaped maxillary permanent lateral incisors:
A meta-analysis. Am J Orthod Dentofacial Orthop 2013;144(1):97-109.

2. Gil Tirlet et Jean Pierre Attal. Le gradient thérapeutique: un concept médical pour les traitements esthétiques; Information Dentaire Nov 2009, $\mathrm{N}^{\circ} 41 / 42 ; 2561-8$.

3. Kim JH, Choi NK, Kim SM. A Retrospective Study of Association between Peg-shaped Maxillary Lateral Incisors and Dental Anomalies. J Clin Pediatr Dent. 2017;41(2):150-53.

4. Ayca Deniz Izgi EA. Direct restorative treatment of peg-shaped maxillary lateral incisors with resin composite: A clinical report. J Prosthet Dent. 2005;93:526-9.

5. Linda G. Treatment options for peg-shaped laterals using direct composite bonding. International Dentistry SA, 12(1); 26-33.

6. Izgi AD, Ayna E. Direct restorative treatment of peg-shaped maxillary lateral incisors with resin composite: a clinical report. J Prosthet Dent. 2005;93(6):526-9.

7. Garber DA. Direct composite veneers versus etched porcelain laminate veneers. Dent Clin North Am. April 1989;33:301-4.

8. Knapp M. Managing the peg lateral incisor. Australian dental association inc. 2013.

9. Schmitz JH, Coffano R, Bruschi A. Restorative and orthodontic treatment of maxillary peg incisors: a clinical report. The Journal of prosthetic dentistry. 2001 Apr 1;85(4):330-4.

10. De Munck JD, Van Landuyt K, Peumans M, Poitevin A, Lambrechts P, Braem M, Van Meerbeek B. A critical review of the durability of adhesion to tooth tissue: methods and results. Journal of dental research. 2005 Feb;84(2):118-32. 